\title{
Use of Rapid Fentanyl Test Strips Among Young Adults Who Use Drugs
}

\author{
Maxwell S. Krieger, BS ${ }^{1}$, William C. Goedel, BA ${ }^{1}$, Jane A. Buxton, MBBS, MHSc, FRCPC ${ }^{2,3}$, \\ Mark Lysyshyn, MD, MPH, FRCPC ${ }^{2,4}$, Edward Bernstein, MD $^{5,6}$, Susan G. Sherman, PhD $^{7}$, \\ Josiah D. Rich, MD, MPH ${ }^{1,8}$, Scott E. Hadland, MD, MPH, MS ${ }^{5,6}$, Traci C. Green, PhD, \\ MSc $^{1,5,6,8}$, Brandon D.L. Marshall, PhD ${ }^{1}$
}

1.Department of Epidemiology, School of Public Health, Brown University, Providence, Rhode Island ${ }^{2}$ School of Population and Public Health, University of British Columbia, Vancouver, Canada ${ }^{3}$.British Columbia Centre for Disease Control, Vancouver, Canada ${ }^{4}$.Vancouver Coastal Health, Vancouver, Canada 5.Department of Emergency Medicine, Grayken Center for Addiction, Boston University School of Medicine, Boston, USA ${ }^{6}$.Department of Pediatrics, Grayken Center for Addiction, Boston Medical Center, Boston, USA 7.Department of Health, Behavior and Society, Johns Hopkins Bloomberg School of Public Health, Baltimore, USA ${ }^{8}$.Department of Emergency Medicine, Warren Alpert Medical School of Brown University, Providence, USA

\section{Abstract}

Background: The overdose epidemic has been exacerbated by a dramatic increase in deaths involving illicitly manufactured fentanyl (IMF). Drug checking is a novel strategy to identify IMF in illicit drugs. We examined the uptake and acceptability of rapid fentanyl test strips among young adults.

Methods: From May to September 2017, we recruited 93 young adults in Rhode Island who reported injecting drugs or using heroin, cocaine, or illicitly obtained prescription pills in the past 30 days. Participants were asked to test either their urine after drug use (post-consumption) or a drug sample prior to use (pre-consumption) using rapid fentanyl test strips. After a questionnaire and a brief training, participants received ten strips for their personal use and were asked to return for a one-month follow-up visit, which assessed the uptake and acceptability of the rapid strips tests, and behavioral outcomes associated with receipt of a positive test.

Results: Of the $81(87 \%)$ participants who returned for follow-up and who had complete data, the mean age was 27, $45(56 \%)$ were male, and 37 (46\%) were non-white. A total of 62

\footnotetext{
Address Correspondence to: Brandon D.L. Marshall, PhD, Associate Professor, Brown University School of Public Health, 121 South Main Street, Box G-S-121-2, Providence, Rhode Island 02912. Telephone: (401) 863-6427. Fax: (401) 863-3173. brandon_marshall@brown.edu.

Author Contributions

MSK helped conduct the surveys, drafted the manuscript, and approved the final manuscript for submission. MSK conducted the statistical analyses, with support from WCG and BDLM. BDLM conceived the pilot study and approved the final manuscript for submission. WCG helped revise the manuscript and assist with critical interpretations of the findings. All authors read the manuscript and revised it critically, providing important intellectual context. All authors approved the final version of the manuscript for submission.

Conflicts of Interest

The authors report no conflicts of interest.
} 
participants (77\%) reported using at least one test strip. Of these, $31(50 \%)$ received at least one positive result. A positive result was associated with older age, homelessness, heroin use, injection drug use, ever witnessing an overdose, and concern about overdose or drugs being laced with fentanyl (all $p<0.05$ ). Receiving a positive result was significantly associated with reporting a positive change in overdose risk behavior between baseline and follow-up ( $p \leq 0.01)$. Among all participants, 79 (98\%) reported confidence in their ability to use the test strips and 77 (95\%) wanted to use them in the future.

Conclusions: Young adults reported high uptake and acceptability of fentanyl test strips to detect IMF in illicit drugs.

\section{Keywords}

Overdose; Opioids; Fentanyl; Harm Reduction; Uptake; Acceptability

\section{Introduction}

The overdose epidemic in the United States (US) has caused an unprecedented number of fatalities over the last decade (Rudd, Aleshire, Zibbell, \& Gladden, 2016). Between 2000 and 2014, the rate of death from drug overdoses has increased by $137 \%$, including a $200 \%$ increase in the rate of overdose deaths involving opioids (Rudd, Aleshire, et al., 2016). Since 2010, deaths due to heroin and synthetic opioids (other than methadone) have increased dramatically in the US, similar to trends observed in Canada, Europe, and Australia (Rudd, Seth, David, \& Scholl, 2016; Seth, Scholl, Rudd, \& Bacon, 2018). Illicitly manufactured fentanyl (IMF), a highly potent synthetic opioid, is increasingly found as an adulterant in the illicit drug supply, or replacing heroin entirely, across the US and in Canada, Europe, and Australia (Fischer, Russell, Murphy, \& Kurdyak, 2015; Mounteney, Giraudon, Denissov, \& Griffiths, 2015; Roxburgh et al., 2013; Somerville, 2017; Tomassoni, 2017). As only a small amount of fentanyl or a fentanyl analogue is needed to result in an overdose, the presence and distribution of IMF throughout the US illicit drug supply poses a significant risk to those unknowingly consuming the substance (Armenian, Vo, Barr-Walker, \& Lynch, 2017; Burns, DeRienz, Baker, Casavant, \& Spiller, 2016; Centers for Disease Control and Prevention, 2015; Daniulaityte et al., 2017; United States Department of Justice, 2015).

As many people who use drugs may be unaware of the presence of IMF in the substances they consume (Macmadu, Carroll, Hadland, Green, \& Marshall, 2017), recent innovations in rapid drug testing have allowed for the use of test strips to detect IMF and prevent fentanylrelated overdose (Ciccarone, 2017; Ciccarone, Ondocsin, \& Mars, 2017; Griswold et al., 2018). Commercially available rapid test strips are able to detect fentanyl, related analogues, and their metabolites in urine (BTNX, 2018). Recently however, these test strips have been used off-label at syringe exchange programs in the US and at a supervised consumption facility in Canada (Bebinger, 2017; Lysyshyn, Dohoo, Forsting, Kerr, \& McNeil, 2017). In these settings, rapid test strips enable people who use drugs to check their drug supply for the presence of fentanyl before consumption by dissolving a small amount of the substance or a sample of drug residue in water (Stewart, 2017). 
Rhode Island is a state that has been severely affected by the opioid epidemic (MercadoCrespo et al., 2014). Despite efforts to reduce overdose mortality (Raimondo, 2015), the state has seen increasing numbers of fentanyl deaths, from 84 deaths in 2014 to 138 in 2016 (Marshall et al., 2017). In Rhode Island, young adults are particularly affected by the presence of fentanyl in the illicit drug supply, with $28 \%$ of fentanyl-related overdose deaths in Rhode Island occurring among this age group (Marshall et al., 2017). This trend mirrors a growing national trend, as young adults are increasingly affected by the fentanyl overdose epidemic (Jones, Logan, Gladden, \& Bohm, 2015; O’Donnell, Halpin, Mattson, Goldberger, $\&$ Gladden, 2017). Young adults may be most affected due to their lack of knowledge concerning harm reduction practices, lack of connection to established harm reduction services, and lower uptake of harm reduction practices (Frank et al., 2015; Yedinak et al., 2016). Suspected IMF exposure is common among people who use drugs in Rhode Island (Macmadu et al., 2017), but demand for fentanyl is low and many wish to avoid it (Carroll, Marshall, Rich, \& Green, 2017).

Previous research reporting baseline results from our pilot study has shown that young adults who use drugs are willing to use rapid fentanyl test strips (Krieger et al., 2018). In addition, some studies have evaluated the uptake and behavioral outcomes associated with fentanyl test strip utilization in supervised consumption settings (Lysyshyn et al., 2017; Tupper, McCrae, Garber, Lysyshyn, \& Wood, 2018). However, there is a paucity of data regarding the feasibility, use, and acceptability of such tests in a real-world, non-supervised setting, particularly among young adults (McGowan, Harris, Platt, Hope, \& Rhodes, 2018). In the current study, we describe the uptake, acceptability, and experiences of using rapid fentanyl test strips among a sample of young adults in Rhode Island who use drugs.

\section{Materials \& Methods}

\subsection{Study Design and Recruitment}

The study design, as well as protocols related to recruitment, survey administration, and fentanyl test strip training and use, have been described previously in detail (Krieger et al., 2018). In brief, we recruited young adults who use drugs in Rhode Island through bus advertisements, online advertisements, fliers, and through word of mouth from May to September 2017. In addition, participants were also recruited from RAPIDS (Rhode Island Young Adult Prescription and Illicit Drug Study), a previous study of young adults in Rhode Island who engaged in non-medical prescription opioid use (Marshall et al., 2018), and who consented to future contact. A total of four RAPIDS participants who were re-contacted and were found to be eligible for the current study were enrolled. Participants were considered eligible for the present study if they: (1) were aged 18 to 35 years old, (2) currently lived in Rhode Island, and (3) self-reported heroin use, cocaine use, injection drug use, or having purchased prescription pills on the street in the past 30 days. These criteria were chosen on the basis of available evidence indicating that fentanyl is contaminating both cocaine and heroin supplies in the US, in addition to being pressed into various counterfeit pills (Centers for Disease Control and Prevention, 2015). We purchased single-use rapid fentanyl urine test strips from BTNX Inc. (Ontario, Canada), which have a detection level of $20 \mathrm{ng} / \mathrm{ml}$ (BTNX, 2018). While there are other fentanyl test strip manufacturers (McKesson, 2018; "PocketLab 
Fentanyl test," 2018), a recent two-site study reported the BTNX fentanyl test strips to be highly sensitive (96 and 100\% at each test site) and specific (90 and 98\%), when compared against laboratory gold standard methods (Bloomberg School of Public Health, 2018). The BTNX test strips were also able to identify fentanyl analogues including acetylfentanyl and furanylfentanyl. However, the rapid fentanyl urine test strips do not indicate the amount of fentanyl or fentanyl analogue present in a sample, only its presence or absence. As the efficacy of these test strips as a harm reduction tool has not yet been established, we conducted a pilot study to determine the feasibility of the intervention to inform a larger trial that would test intervention efficacy. This pilot study was approved by the Brown University Institutional Review Board, which was a distinct IRB application from our previous study, RAPIDS.

Eligible participants agreed to two visits. The first visit included an interviewer-administered survey and a short training on how to use the rapid fentanyl test strips. The baseline survey asked questions about socio-demographic characteristics, drug use behaviors, drug use history, and attitudes towards and experiences with fentanyl. The training included watching a brief video on using the test strips for either urine or drugs followed by another video which described interpreting the test results. Participants were allowed to ask questions at any time and were given a test strip during the training to examine. At the end of the survey and training, participants received 10 rapid fentanyl test strips for personal use before their next visit, as well as written materials describing how to use the test strips by testing their urine or by testing drug residue from a baggie or by dissolving a small amount of the substance in a cooker (depending on their assigned testing method, see below). The materials also included written and graphical information showing how to interpret a test result. In addition, each individually packaged test strip included a sticker which showed a positive and negative result, with the language "fentanyl" for a positive result and "unknown" for a negative result.

In addition to training on using and interpreting the rapid fentanyl test strips, participants also received overdose education and naloxone distribution (OEND). Overdose prevention education included information on how to safely use drugs (such as not drugs using alone, keeping naloxone nearby, and titrating doses), as well as materials on how to recognize and respond to an overdose. Participants also received naloxone training along with a $4 \mathrm{mg}$ intranasal naloxone kit to take home. Participants were subsequently asked if they had any questions or concerns about any of the aforementioned training materials. All naloxone training and overdose education were administered by trained research staff. The training protocol and other materials are available upon request.

For the purposes of this pilot study, we sought to determine the feasibility of both urine testing and drug sample testing, given that the latter is currently an off-label use of the device. The first 40 participants were assigned to a "post-consumption" group and were given instructions on how to test their urine (after drug consumption) using the rapid fentanyl test strips. After 40 participants were recruited, remaining participants were assigned to a "pre-consumption" testing group, and were instructed on how to test drug residue or a drug sample (prior to consumption) using the rapid fentanyl test strips. To mitigate the risk associated with receiving a false negative test result, participants were 
informed that fentanyl or fentanyl analogues may still be present in urine or a drug sample despite a negative test strip result. All participants were instructed to engage in overdose prevention behaviors (described above) regardless of the test result. A minimum of two weeks after their first visit, participants were contacted using their preferred contact method (text, call, or email) to schedule a second visit. During the second visit, participants completed a short interview-administered survey which asked questions about their experiences using the rapid fentanyl test strips, as well as similar questions about perceptions of fentanyl from the first survey. In total, 93 participants completed a baseline interview and $83(89.2 \%)$ returned for a follow-up interview. Two participants were excluded from analysis because of missing information, resulting in an analytic sample of $n=81$. Participants were compensated $\$ 25$ for the first visit and $\$ 50$ for the second visit.

\subsection{Measures}

This study had two primary outcomes of interest. First, at the follow-up visit, participants were asked if they used any of the strip tests (yes vs. no). Next, among those who reported using at least one strip test, participants were asked to report how many were positive. The second primary outcome was operationalized by categorizing participants into those who reported at least one positive test (yes vs. no).

A variety of baseline demographic and behavioral characteristics served as the independent variables of interest. These included gender, race, ethnicity, education, homelessness, regular drug use (defined as at least once a week), and lifetime history of having ever injected drugs, dealt drugs, overdosed, or witnessed an overdose. In addition, we examined various attitudes and experiences related to fentanyl at baseline as potential predictors of strip test utilization and receipt of at least one positive test at follow-up. Specifically, we asked participants at their first visit about their attitudes about overdose and fentanyl with prompts such as "I am concerned about my drugs being contaminated with fentanyl" and "I am concerned about overdosing". Response options were offered on a 4-point Likert scale of strongly agree to strongly disagree. Variables were recoded into two categories: "agree" (for answers strongly agree or agree), and "disagree/neutral" (for answers strongly disagree, disagree, do not know or refuse). We also assessed fentanyl-related experiences with questions such as "Have you ever used a drug you were confident was laced with fentanyl?"

In a secondary analysis, we also sought to determine the extent of behavior change following receipt of a positive test result. At the first visit, participants were asked about previous behaviors related to suspected fentanyl exposure through multiple choice questions including, "The last time you used a drug you were confident was laced with fentanyl, what did you do when you found out you had drugs laced with fentanyl?" and for participants who were unsure if they had ever used fentanyl-laced drugs, "What would you do if you found out your drugs had fentanyl in them?" To determine behavior change among participants who reported receiving a positive test result at the second visit, answers to the aforementioned questions were compared with answers to the follow-up question, "What did you do after you found out you had drugs laced with fentanyl?". Answers to the baseline and follow-up questions included the following risk reduction practices: "Did a tester", "Went slower", "Threw them out", "Used less", and "Used with someone else around". Other 
response options included "Gave them away", "Sell them", and "Took them anyways". Participants who did not report at least one positive risk reduction behavior at baseline but who did after receiving a positive test result were considered to have had "Any positive overdose risk behavior change related to a fentanyl test result" (yes vs. no).

Finally, participants were asked a number of questions at follow-up to ascertain the feasibility, acceptability, and willingness to continue to use the strip tests. These questions included "I feel confident in my ability to read the results of the fentanyl testing strips", "It would be easy to continue to use the fentanyl rapid tests", and "I would like to use fentanyl rapid tests in the future". Response options were offered on a four-point Likert scale but were recoded as "agree" or "disagree/neutral".

\subsection{Statistical Analyses}

First, we calculated descriptive statistics for all key study variables. Second, we assessed bivariable associations of various socio-demographic, behavioral, attitudinal, and experiential factors associated with: (1) use of at least one rapid fentanyl test strip among all participants, and (2) receipt of at least one positive test result among participants who used at least one rapid fentanyl test strip. Pearson's chi-square test and the Wilcoxon rank sum test were used to compare categorical and continuous variables, respectively. Fisher's exact test was used in cases where cell counts were less than 5. Two-sided $p$ values were used for all variables and were considered statistically significant at 0.05 .

\section{Results}

A total of 93 participants completed the baseline interview. The analytical sample includes participants who completed the baseline interview, returned for a follow-up interview, and who had complete outcome data $(n=81,87 \%)$. There were no significant demographic or relevant behavioral differences between participants who returned for the second interview and those who were lost to follow-up (results not shown). Of the 81 participants who returned for follow-up and constituted the analytic sample, 34 participants were instructed to use the test strips to test their urine post-consumption (42\%), and 47 participants were instructed to use the test strips to test drug residue or a sample of their drugs preconsumption (58\%). At baseline, a significantly greater number of participants used cocaine at least once a week in the pre-consumption testing group (49\%) compared to the postconsumption testing group $(24 \%, p=.022)$.

The demographic and behavioral characteristics of the analytical sample are shown in Table 1 , and the fentanyl-related attitudes and experiences of participants are summarized in Table 2. The average age in the analytic sample was 26.5 years (standard deviation [SD]: 4.7). More than half of the sample identified as male (56\%), and 37 (46\%) were non-white. About one-quarter identified as being of Hispanic or Latino descent (24\%). Over half had ever been homeless (59\%). One-third of participants used heroin at least once a week (33\%), and approximately half of the sample had ever injected drugs (52\%). One-third had ever experienced an overdose (36\%), and two-thirds had ever witnessed an overdose (62\%). Most agreed with the statement that they were concerned about their drugs being contaminated 
with fentanyl (68\%), about their friends using drugs contaminated with fentanyl (79\%), and about their friends overdosing $(73 \%)$.

At follow-up, most participants $(n=62,77 \%)$ reported using at least one of the provided rapid fentanyl test strips. Participants used a median of 3 rapid test strips (interquartile range [IQR]: $1-6)$. A total of 10 participants $(12 \%, n=4$ from pre-consumption and $n=6$ from postconsumption) used all ten provided test strips. Of note, about half of the participants (58\%) gave test strips to others. Rapid test strip utilization was more common among participants who had reported ever dealing drugs at baseline $(p=.038)$. There were no additional statistically significant differences in demographic characteristics (see Table 1) or in fentanyl-related attitudes and experiences (Table 2) between those who used the rapid test strips and those who did not.

Among those who used at least one test strip $(n=62), 31(50 \%)$ received at least one positive result. There were no significant differences in receipt of a positive result between the two testing method groups (results not shown), but there were a number of significant differences based on demographic and behavioral factors (Table 1), as well as fentanylrelated attitudes and experiences (Table 2). Specifically, individuals who received a positive test result were older on average $(p=.030)$ and a greater number had ever been homeless ( $p$ $=.040)$, used heroin at least once a week $(p<.001)$, ever injected drugs $(p<.001)$, and ever witnessed an overdose $(p<.001)$. In addition, almost all individuals who received a positive test result were also concerned about their friends using drugs contaminated with fentanyl at baseline ( $p=.001)$, about their friends experiencing an overdose at baseline $(p<.001)$, and about experiencing an overdose themselves at baseline $(p=.001)$. A majority of individuals who received a positive test result also reported ever using a drug that they suspected was contaminated with fentanyl at baseline $(p<.001)$ or to have reported ever experiencing an overdose due to a drug that they suspected was contaminated with fentanyl at baseline ( $p=$. 022).

Participants reported a number of actions after receiving a positive test result. Among those who received a positive test result after testing their drugs or urine $(n=31), 45 \%$ used smaller amounts, $42 \%$ went slower when using, $39 \%$ used with someone else, and $36 \%$ did a tester. Few participants threw their drugs out (10\%), sold them (10\%), or gave them away (7\%). Among participants who used at least one strip, receipt of a positive test result was associated with reporting a positive overdose risk behavior change between the baseline and follow up visit ( $p \leq .001$, see Table 3 ). No significant differences in behavior change were observed between participants who used at least one fentanyl test strips and those who did not (see Table 3). Between the pre-consumption and post-consumption testing groups, a greater proportion of those from the pre-consumption group reported a positive behavior change after receipt of a positive test result (62\% vs. $38 \%$, respectively), such as using smaller amounts or using with someone else around. There were no other notable differences between testing groups (data not shown).

At follow-up, nearly all participants $(n=79,98 \%)$ reported confidence in their ability to use the test strips to test their urine or drugs for the presence of fentanyl. In addition, almost all participants reported wanting to continue using the test strips in the future $(n=77,95 \%)$, and 
stated that it would be easy to continue to use the strips ( $\mathrm{n}=75,93 \%)$. A larger number of participants who used at least one rapid fentanyl test strip reported wanting to use rapid tests in the future ( $p=0.04)$ compared to those who had not used any rapid tests (see Table 3 ). Participants also reported that they felt confident in their ability to read the results of the fentanyl testing strips $(n=62,77 \%)$. Participants reported being comfortable obtaining test strips at a diverse range of potential distribution locations, including community health clinics (72\%), community-based organizations (57\%), pharmacies (52\%), health department offices $(52 \%)$, needle and syringe exchange programs (49\%), and online stores (44\%).

\section{Discussion}

To our knowledge, this is among the first studies to provide proof of concept for the use of rapid fentanyl test strips as a novel harm reduction strategy among people who use drugs. Our previous research reporting baseline results from the same pilot project showed high willingness to use rapid fentanyl test strips among young adults who use drugs (Krieger et al. 2018). In this follow-up study, we observed high uptake and acceptability of rapid fentanyl test strips, as well as high reported confidence in the ability to use the tests, regardless of whether participants were instructed to test a sample of their urine postconsumption or sample of their drug supply or drug residue pre-consumption. The vast majority of individuals reported wanting to continue using the tests in the future, and stated that it would be easy to continue using them. Taken together, these results suggest that rapid fentanyl test strips may be an acceptable intervention among young adults who use drugs in this setting. Our results also highlight the further need for research assessing the efficacy and effectiveness of rapid fentanyl test strip use on uptake of effective harm reduction practices, particularly when implemented in combination with other educational and behavior change intervention strategies aimed at decreasing risk of overdose among people who use drugs.

Among those who used at least one test strip, half received at least one positive test result, suggesting that IMF exposure may be commonplace among young people in Rhode Island who regularly use heroin, cocaine, and counterfeit prescription pills. Many of the demographic and behavioral factors most strongly associated with receipt of a positive test (i.e., injection drug use, frequent heroin use) were also identified as correlates of recent IMF exposure in a recent urine toxicology screening study among people who use drugs in Vancouver, Canada (Hayashi et al., 2018). Those who received a positive test result were also associated with being concerned about their friends using drugs contaminated with fentanyl and about their friends experiencing overdose. Notably, although not explicitly encouraged by our research staff, a high number of participants distributed their test strips to others. Further research is needed to understand how social and drug-using networks could be leveraged for rapid dissemination of rapid fentanyl test strips and other novel strategies (e.g., incorporating fentanyl testing interventions into existing OEND programs) to reduce risk of overdose among young adults.

Study participants reported that they would feel most comfortable obtaining rapid fentanyl test strip kits at community health clinics and community-based organizations. These results suggest that initial distribution of rapid fentanyl test strips could be deployed to this population through organizations that currently offer other harm reduction services. For 
example, community-based organizations that conduct overdose prevention education and outreach efforts may be ideal venues for rapid fentanyl test strip training and distribution, as these organizations also distribute other harm reduction supplies (e.g., naloxone, sterile needles and syringes).

Finally, receipt of a positive test result was associated with positive risk reduction behavior change (e.g., using less of the substance, consuming with others around) compared to behaviors reported at the baseline visit. This finding serves as preliminary evidence that fentanyl test strips could promote engagement in risk reduction practices, which may in return reduce rates of overdose among young people who use drugs. An ideal implementation strategy would be to couple rapid fentanyl test strip distribution with OEND programs, which would include information about steps to take after receipt of a positive test result. A larger study that assesses the potential effectiveness of fentanyl testing trips on overdose and overdose risk behavior is warranted.

These findings are not without limitations. First, this study relied on self-reports and thus is limited by biases including social desirability and recall. In addition, we did not verify or confirm reported test strip results. Second, the study represents a convenience sample of young adults in Rhode Island who use drugs. Although our sample may represent those at high risk for future overdose (as one-third had ever previously overdosed), these findings may not be generalizable to older populations of people who use drugs, or in other regions of the United States outside of New England. In addition, Rhode Island is severely affected by the fentanyl overdose epidemic, and findings may not be generalizable to other regions of the country in which IMF is not as prevalent. Third, the small sample size of our study may not be generalizable to larger populations. Additionally, the limited sample size impeded our ability to conduct multivariable analyses. As this was a small pilot study to provide proof of concept for the use of rapid fentanyl test strips, a larger efficacy study is warranted. Fourth, given that pre-consumption drug testing via rapid fentanyl test strips represents off-label use of the study product, further research is needed to assess the sensitivity and specificity of these test strips in detecting fentanyl and its related analogues in drug samples when used by people who use drugs, compared to traditional laboratory-based methods. Fifth, we did not assess reasons for not using the tests among the minority (23\%) who reported not using the strips at follow-up. Further research is needed to determine why some participants may not engage in pre- or post-consumption drug checking in settings where the technology is available. Finally, further research is needed to test the efficacy of the use of these test strips in combination with a behavioral intervention to increase use of established harm reduction practices, increase risk reduction behaviors, and prevent opioid overdose. Nonetheless, our findings demonstrate the acceptability and feasibility of rapid fentanyl test strips to detect IMF presence in illicit drugs among young people who use drugs.

\section{Conclusions}

In this study, young adults using drugs in Rhode Island reported high uptake and acceptability of rapid fentanyl test strips, suggesting that rapid fentanyl test strips are a feasible and acceptable harm reduction tool to detect IMF presence in illicit drugs. 


\section{Acknowledgements}

We would like to thank the study participants for their contribution to the research, as well as our researchers, students, and staff, including Jacqueline Goldman, Conor Millard, Kobe Pereira, Giovannia Barbosa, and Esther Manu.

Role of Funding Source

This project was supported by a research seed grant from the Office of the Vice President of Research at Brown University to Brandon D.L. Marshall, PhD. The funders had no role in study design, data analysis, or decision to publish. The views expressed in this manuscript do not reflect the opinions of the funder.

\section{References}

Armenian P, Vo KT, Barr-Walker J, \& Lynch KL (2017). Fentanyl, fentanyl analogs and novel synthetic opioids: A comprehensive review. Neuropharmacology. 10.1016/j.neuropharm. 2017.10.016

Bebinger M (2017, May 11). As Fentanyl Deaths Rise, An Off-Label Tool Becomes A Test For The Killer Opioid. Retrieved January 25, 2018, from http://www.wbur.org/commonhealth/2017/05/11/ fentanyl-test-strips

Bloomberg School of Public Health. (2018). Fentanyl Overdose Reduction Checking Analysis Study. Retrieved August 9, 2018, from https://americanhealth.jhu.edu/fentanyl

BTNX. (2018, February 6). Rapid Response Fentanyl (FYL) Test Strip. Retrieved October 18, 2017, from http://www.btnx.com/Product?id=2002

Burns G, DeRienz RT, Baker DD, Casavant M, \& Spiller HA (2016). Could chest wall rigidity be a factor in rapid death from illicit fentanyl abuse? Clinical Toxicology, 54(5), 420-423. [PubMed: 26999038]

Carroll JJ, Marshall BDL, Rich JD, \& Green TC (2017). Exposure to fentanyl-contaminated heroin and overdose risk among illicit opioid users in Rhode Island: A mixed methods study. The International Journal on Drug Policy, 46, 136-145. [PubMed: 28578864]

Centers for Disease Control and Prevention. (2015). Increases in Fentanyl Drug Confiscations and Fentanyl-related Overdose Fatalities (No. CDCHAN-00384). Centers for Disease Control and Prevention Retrieved from https://emergency.cdc.gov/han/han00395.asp

Ciccarone D (2017). Fentanyl in the US heroin supply: A rapidly changing risk environment. The International Journal on Drug Policy, 46, 107-111. [PubMed: 28735776]

Ciccarone D, Ondocsin J, \& Mars SG (2017). Heroin uncertainties: Exploring users' perceptions of fentanyl-adulterated and -substituted "heroin." The International Journal on Drug Policy, 46, 146155. [PubMed: 28735775]

Daniulaityte R, Juhascik MP, Strayer KE, Sizemore IE, Harshbarger KE, Antonides HM, \& Carlson RR (2017). Overdose deaths related to fentanyl and its analogs - Ohio, January-February 2017. MMWR. Morbidity and Mortality Weekly Report, 66(34), 904-908. [PubMed: 28859050]

Fischer B, Russell C, Murphy Y, \& Kurdyak P (2015). Prescription opioids, abuse and public health in Canada: is fentanyl the new centre of the opioid crisis? Pharmacoepidemiology and Drug Safety, 24(12), 1334-1336. [PubMed: 26502744]

Frank D, Mateu-Gelabert P, Guarino H, Bennett A, Wendel T, Jessell L, \& Teper A (2015). High risk and little knowledge: overdose experiences and knowledge among young adult nonmedical prescription opioid users. The International Journal on Drug Policy, 26(1), 84-91. [PubMed: 25151334]

Griswold MK, Chai PR, Krotulski AJ, Friscia M, Chapman B, Boyer EW, ... Babu KM (2018). Selfidentification of nonpharmaceutical fentanyl exposure following heroin overdose. Clinical Toxicology, 56(1), 37-42. [PubMed: 28681615]

Hayashi K, Milloy M-J, Lysyshyn M, DeBeck K, Nosova E, Wood E, \& Kerr T (2018). Substance use patterns associated with recent exposure to fentanyl among people who inject drugs in Vancouver, Canada: A cross-sectional urine toxicology screening study. Drug \& Alcohol Dependence, 183, 16. [PubMed: 29220642] 
Jones CM, Logan J, Gladden RM, \& Bohm MK (2015). Vital Signs: Demographic and Substance Use Trends Among Heroin Users - United States, 2002-2013. MMWR. Morbidity and Mortality Weekly Report, 64(26), 719-725. [PubMed: 26158353]

Krieger MS, Yedinak JL, Buxton JA, Lysyshyn M, Bernstein E, Rich JD, ... Marshall BDL (2018). High willingness to use rapid fentanyl test strips among young adults who use drugs. Harm Reduction Journal, 15(1), 7. [PubMed: 29422052]

Lysyshyn M, Dohoo C, Forsting S, Kerr T, \& McNeil R (2017). Evaluation of a fentanyl drug checking program for clients of a supervised injection site, Vancouver, Canada Presented at the Harm Reduction International, Montreal, Canada Retrieved from https://www.hri.global/abstracts/ abstrct/188

Macmadu A, Carroll JJ, Hadland SE, Green TC, \& Marshall BDL (2017). Prevalence and correlates of fentanyl-contaminated heroin exposure among young adults who use prescription opioids nonmedically. Addictive Behaviors, 68(Supplement C), 35-38. [PubMed: 28088741]

Marshall BDL, Green TC, Elston B, Yedinak JL, Hadland SE, \& Clark MA (2018). The Effectiveness of Internet- and Field-Based Methods to Recruit Young Adults Who Use Prescription Opioids Nonmedically. Substance Use \& Misuse, 1-12.

Marshall BDL, Krieger MS, Yedinak JL, Ogera P, Banerjee P, Alexander-Scott NE, ... Green TC (2017). Epidemiology of fentanyl-involved drug overdose deaths: A geospatial retrospective study in Rhode Island USA., The International Journal on Drug Policy, 46, 130-135. [PubMed: 28601512]

McGowan CR, Harris M, Platt L, Hope V, \& Rhodes T (2018). Fentanyl self-testing outside supervised injection settings to prevent opioid overdose: Do we know enough to promote it? The International Journal on Drug Policy, 58, 31-36. [PubMed: 29758542]

McKesson. (2018). Drugs of Abuse Test Single Drug FEN (Fentanyl) Urine Sample. Retrieved August 7, 2018, from https://mms.mckesson.com/product/1060682/Healgen-Scientific-Ltd-HDFN-114

Mercado-Crespo MC, Sumner SA, Spelke MB, Sugerman DE, Stanley C, \& EIS officer CDC. (2014). Notes from the field: increase in fentanyl-related overdose deaths - Rhode Island, November 2013March 2014. MMWR. Morbidity and Mortality Weekly Report, 63(24), 531. [PubMed: 24941333]

Mounteney J, Giraudon I, Denissov G, \& Griffiths P (2015). Fentanyls: Are we missing the signs? Highly potent and on the rise in Europe. The International Journal on Drug Policy, 26(7), 626-631. [PubMed: 25976511]

O’Donnell JK, Halpin J, Mattson CL, Goldberger BA, \& Gladden RM (2017). Deaths Involving Fentanyl, Fentanyl Analogs, and U-47700 - 10 States, July-December 2016. MMWR. Morbidity and Mortality Weekly Report, 66(43), 1197-1202. [PubMed: 29095804]

PocketLab Fentanyl test. (2018). Retrieved August 22, 2018, from https://www.exchangesupplies.org/ shopdisp_Sure_Screen_PocketLab_Fentanyl_Powder_Test.php

Raimondo GGM (2015). Overdose Prevention and Intervention Task Force. Retrieved April 6, 2018, from http://www.governor.ri.gov/initiatives/odtaskforce/

Roxburgh A, Burns L, Drummer OH, Pilgrim J, Farrell M, \& Degenhardt L (2013). Trends in fentanyl prescriptions and fentanyl-related mortality in Australia. Drug and Alcohol Review, 32(3), 269275. [PubMed: 23442164]

Rudd RA, Aleshire N, Zibbell JE, \& Gladden RM (2016). Increases in Drug and Opioid Overdose Deaths--United States, 2000-2014. MMWR. Morbidity and Mortality Weekly Report, 64(50-51), 1378-1382. [PubMed: 26720857]

Rudd RA, Seth P, David F, \& Scholl L (2016). Increases in Drug and Opioid-Involved Overdose Deaths - United States, 2010-2015. MMWR. Morbidity and Mortality Weekly Report, 65(5051), 1445-1452. [PubMed: 28033313]

Seth P, Scholl L, Rudd RA, \& Bacon S (2018). Overdose Deaths Involving Opioids, Cocaine, and Psychostimulants - United States, 2015-2016. MMWR. Morbidity and Mortality Weekly Report, 67(12), 349-358. [PubMed: 29596405]

Somerville NJ (2017). Characteristics of Fentanyl Overdose — Massachusetts, 2014-2016. MMWR. Morbidity and Mortality Weekly Report, 66 10.15585/mmwr.mm6614a2 
Stewart B (2017, November 28). Demand for fentanyl test strips booms — but test is not widely available. Retrieved November 29, 2017, from http://www.cbc.ca/news/health/demand-forfentanyl-test-strips-booms-but-test-is-not-widely-available-1.4420625

Tomassoni AJ (2017). Multiple Fentanyl Overdoses — New Haven, Connecticut, June 23, 2016. MMWR. Morbidity and Mortality Weekly Report, 66 10.15585/mm6604a4

Tupper KW, McCrae K, Garber I, Lysyshyn M, \& Wood E (2018). Initial results of a drug checking pilot program to detect fentanyl adulteration in a Canadian setting. Drug and Alcohol Dependence, 190, 242-245. [PubMed: 30064061]

United States Department of Justice. (2015, March 18). DEA Issues Nationwide Alert on Fentanyl as Threat to Health and Public Safety. Retrieved October 13, 2017, from https://www.dea.gov/ divisions/hq/2015/hq031815.shtml

Yedinak JL, Kinnard EN, Hadland SE, Green TC, Clark MA, \& Marshall BDL (2016). Social context and perspectives of non-medical prescription opioid use among young adults in Rhode Island: A qualitative study. The American Journal on Addictions / American Academy of Psychiatrists in Alcoholism and Addictions, 25(8), 659-665. 


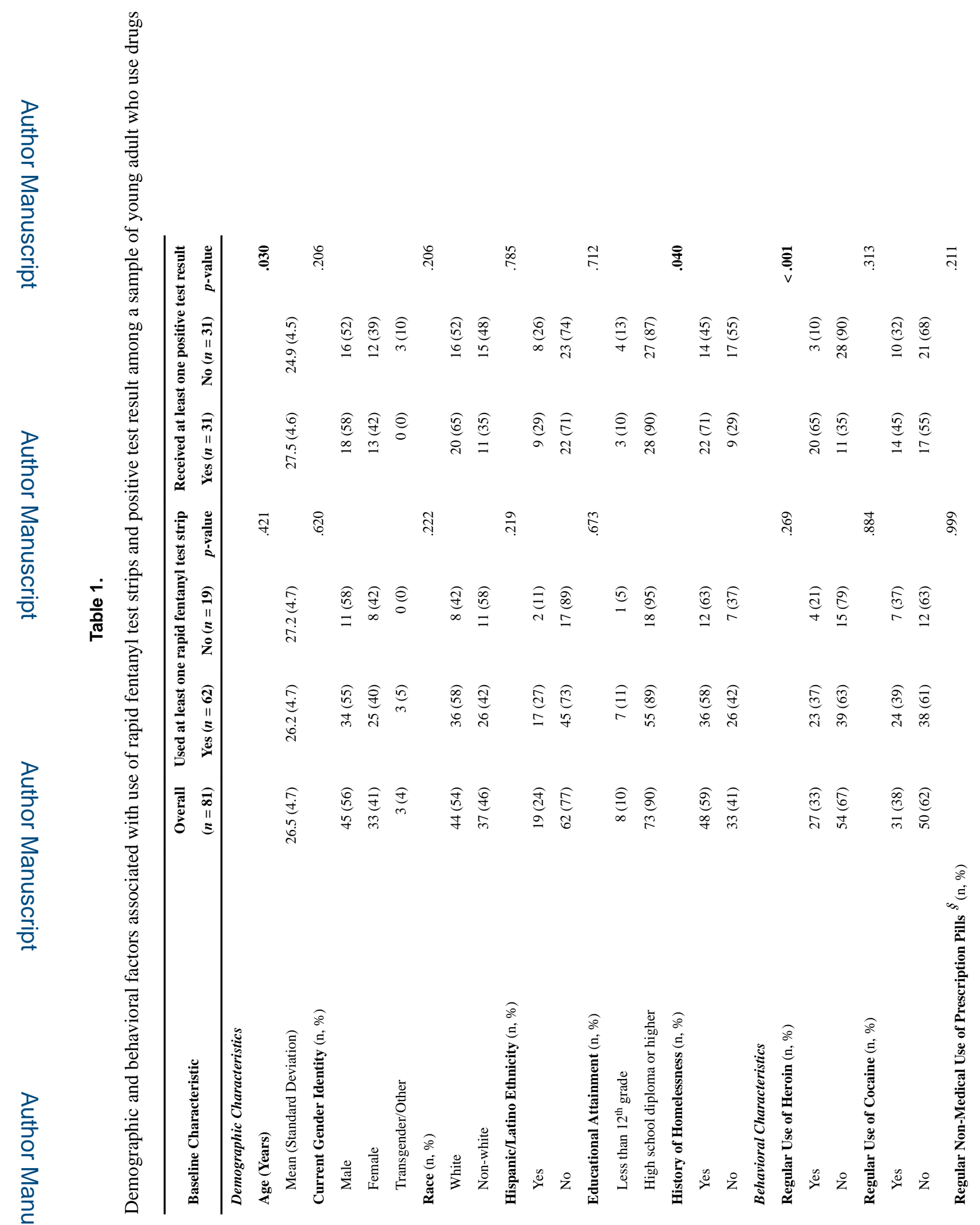

Int J Drug Policy. Author manuscript; available in PMC 2019 November 01. 
Krieger et al.

Page 14

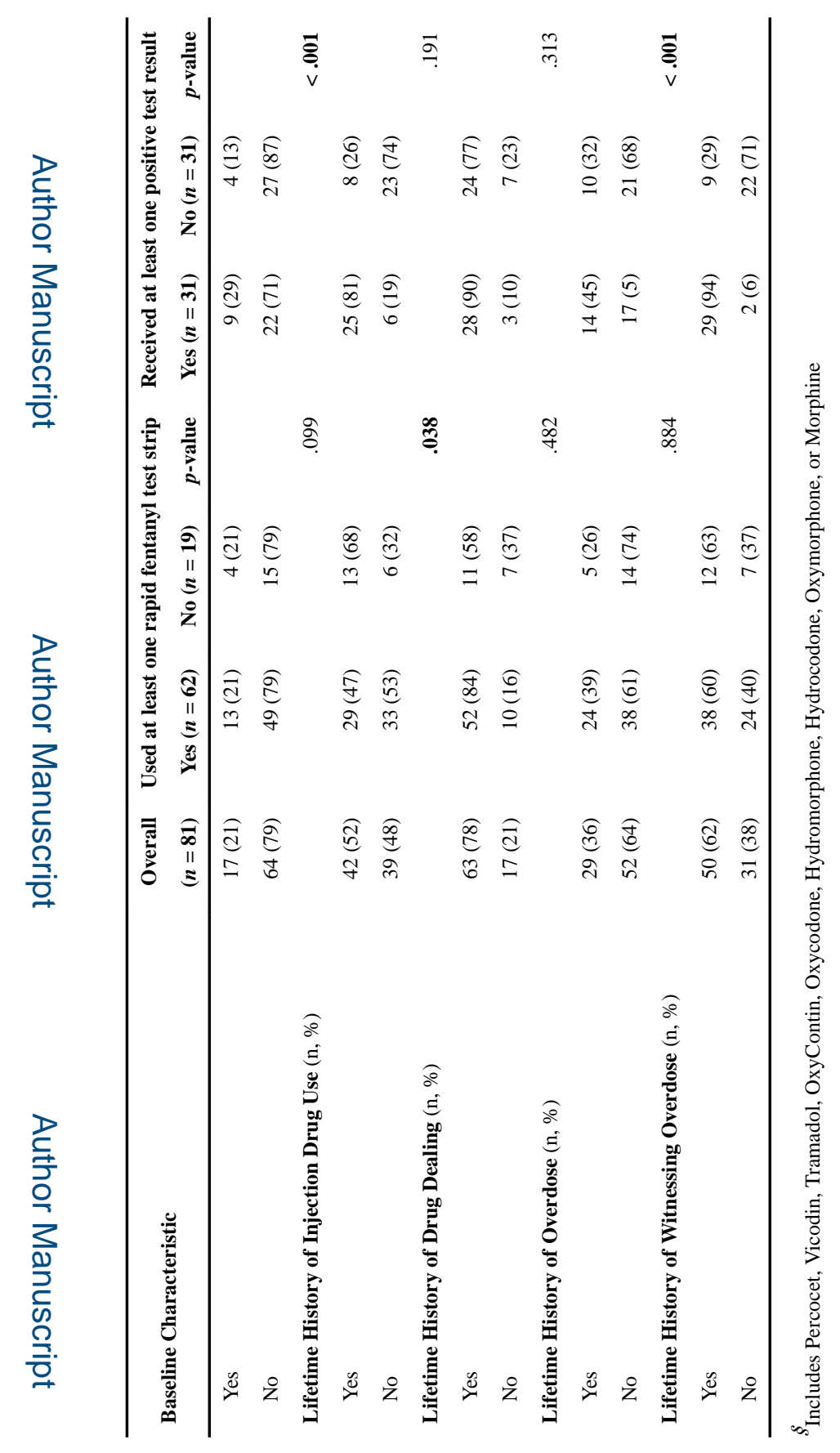

로을

Int J Drug Policy. Author manuscript; available in PMC 2019 November 01. 


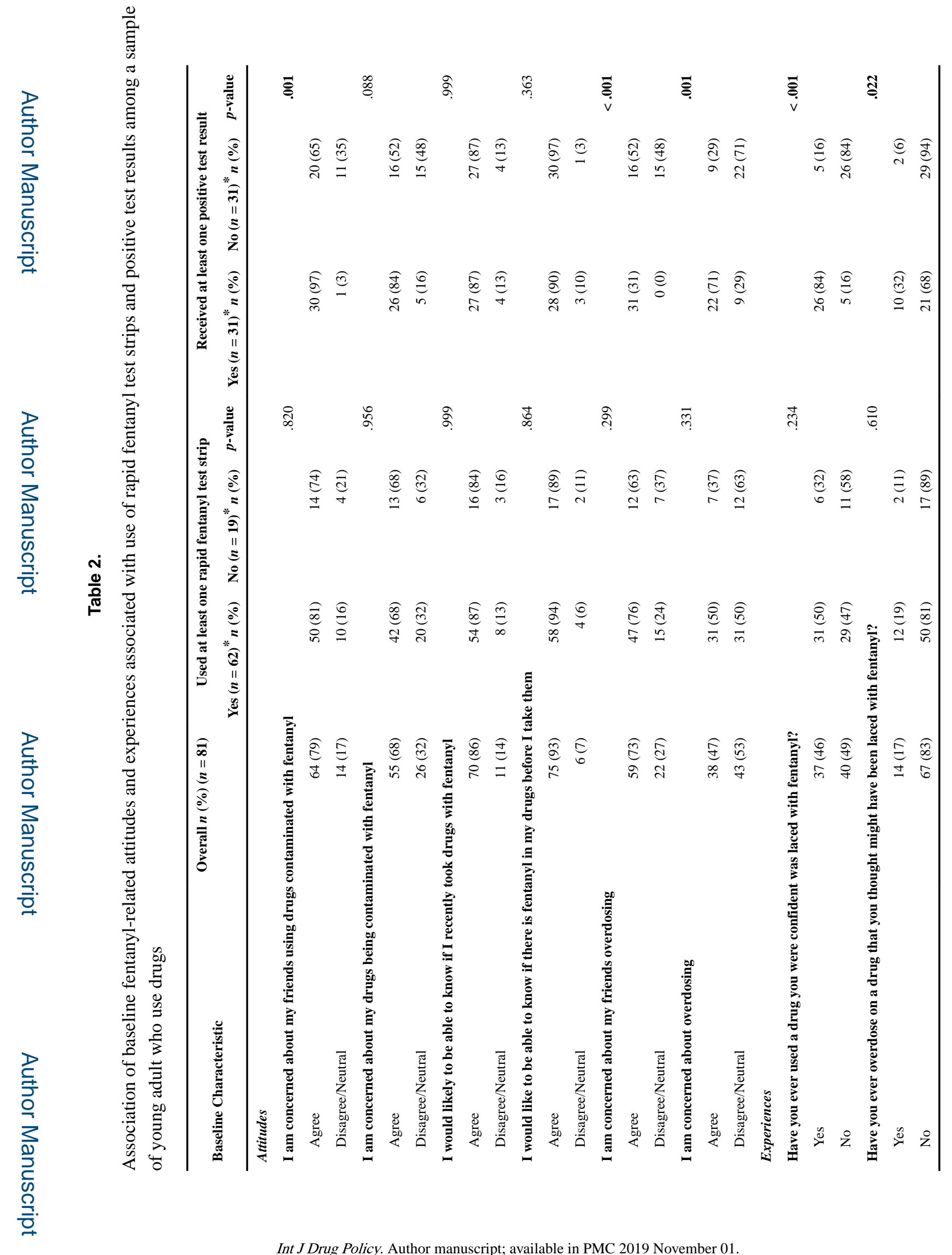




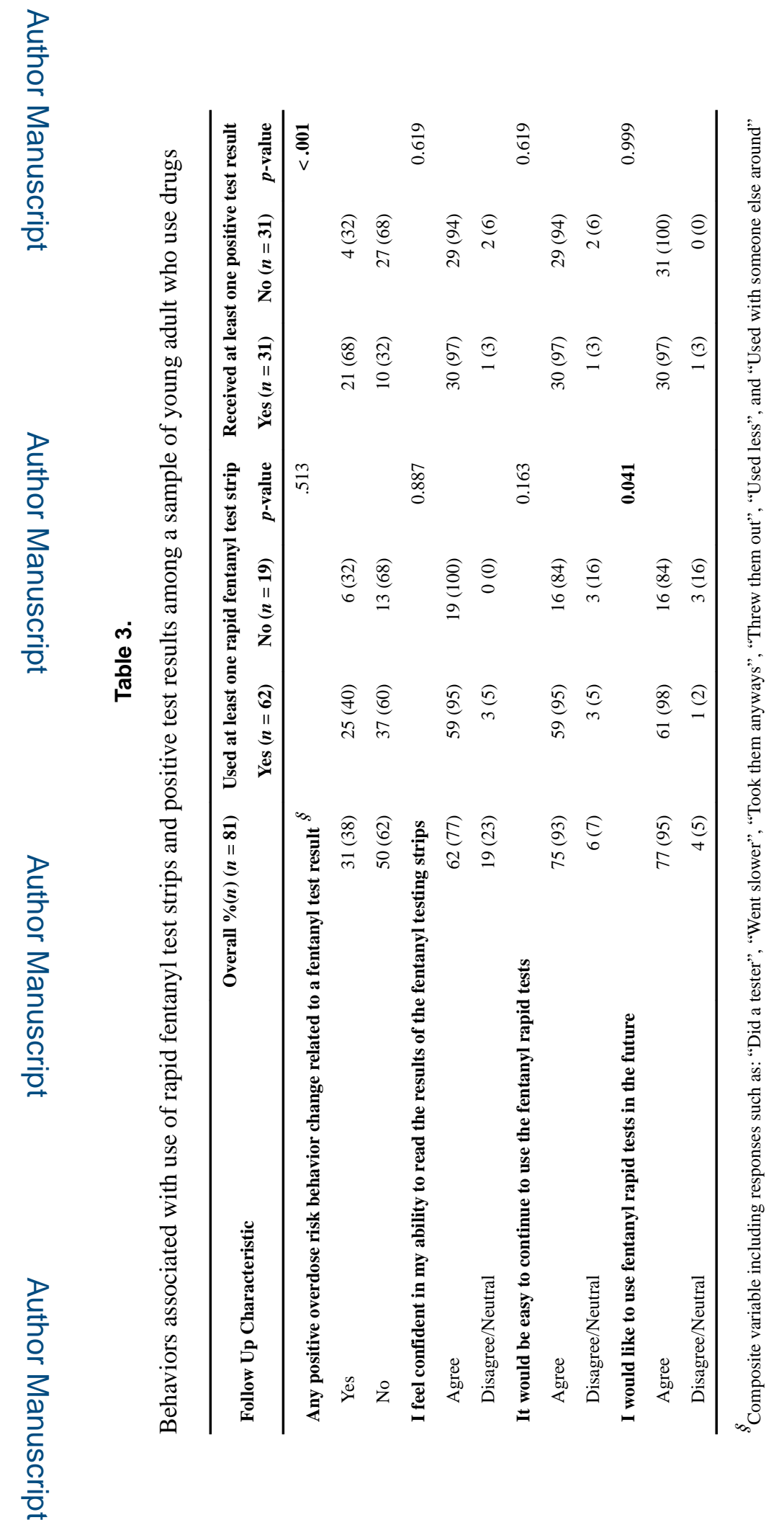

Int J Drug Policy. Author manuscript; available in PMC 2019 November 01. 